\title{
Wie fremd ist uns das Fremde?
}

\author{
Dr. phil. Olga Rösch
}

\section{Vorwort}

In der Literatur zur interkulturellen Kommunikation ist oft die Rede von sprach- und kulturbedingten Mißverständnissen, die daraus resultieren, daß man das Fremde nicht „richtig“" verstanden hat.

- Kann das Fremde überhaupt bis zu Ende „richtig“ verstanden werden?

- Welcher ist der „richtige“ Weg, das Fremde zu verstehen und zu internalisieren?

- Bleibt das verstandene Fremde immer noch fremd oder wird es zum Eigenen?

- Wie scharf ist die Grenze zwischen dem Fremden und dem Eigenen?

Das sind alles theoretisch-methodologische und praktische Fragen zugleich. Sie sind kaum erschöpfend und eindeutig zu beantworten, was aber nicht heißen kann, daß nicht nach den Antworten gesucht wurde und wird. Auf jede Frage kann es überdies mehrere „richtige“ Antworten geben (die eines Psychologen oder eines Theologen usw.), was die Vielschichtigkeit der Problematik andeutet.

\section{Welches Fremde meinen wir?}

Der Erforschung des Fremden widmen sich - wie oben kurz erwähnt - mehrere wissenschaftliche Disziplinen: die Fremdsprachenphilologien, die Ethnologie, Soziologie, Psychologie, Kulturwissenschaft u. a. Entsprechend differieren hier Forschungsziele, Fragestellungen und auch die Definitionen des Fremden. Von der Aufzählung der letzteren möchte ich hier absehen. Obwohl im Kontext unserer Tagung kaum Mißverständnisse in bezug auf den Begriff „Fremder“ auftreten können, will ich es dennoch explizit verdeutlicht wissen, daß wir uns im großen und ganzen der kulturanthropologischen Definition des Fremden anschließen. In der Kulturanthropologie wird ein Mensch als Fremder angesehen, wenn er Träger einer anderen Sprache und Kultur ist, d. h. einer anderen Kulturgemeinschaft angehört.

Das Fremde als Forschungsthema ist bei weitem nicht neu, auch wenn es sehr aktuell erscheinen mag. Bereits seit Beginn unseres Jahrhunderts ist es in den Wissenschaften präsent. Anfang der 70er Jahre erfährt die Fremdheitsforschung einen bemerkenswerten Konjunkturaufschwung, weil die Wirtschaft ihren Bedarf an derartigem Wissen angemeldet hat (Wierlacher, 1993:1938). Inzwischen sprechen wir von einer interdisziplinären wissenschaftlichen Xenologie, der Wissenschaft vom Fremden ${ }^{1}$. Abnehmer der xenologischen Forschungsergebnisse sind heutzutage nicht nur die staatlichen Bibliotheken, sondern auch internationale Unternehmen, die in die Weiterbildung ihrer Mitarbeiter investieren, d. h. interkulturelle Trainings durchführen.

Im Unterschied zur Wissenschaft kann die schöngeistige Literatur auf eine längere Tradition der Behandlung des Themas des Fremden zurüickblicken. Das Motiv des Fremden ist dort so alt wie die europäischen Nationalliteraturen selbst. Man denke nur an die Odyssee von Homer oder an die umfangreiche Reiseliteratur zu allen Zeiten. Das Fremde wird dort stets als Faszination und Bedrohung ${ }^{2}$ beschrieben. Das Fremde weckt Neugier und birgt Gefahren, das Fremde bringt uns Wissenszuwachs und bewegt uns zur Rückkehr in das aus der Ferne so geliebte Eigene (das bekannte Heimkehrmotiv in der Literatur).

Die Begegnung mit der Fremde: Empfinden wir heute zweieinhalb Jahrtausende nach Odysseus - die Fremde emotional grundsätzlich anders?

Doch zurück zu der Frage, die hier als Thema gewählt wurde: Wie fremd ist uns das Fremde? Unsere Fragestellung ist weder eine ideologische, noch eine psychologische und auch keine rein kulturtheoretische. Sie läuft in erster Linie auf kommunikative Aspekte der Begegnung mit dem Fremden hinaus. Hier sei uns eine leichte Paraphrase des bekannten Satzes „Man kann nicht nicht kommunizieren." des Psychologen Paul Wazlawick im Sinne der interkulturellen Kommunikation erlaubt: Auch in einer fremdkulturellen Umgebung kann man nicht nicht kommunizieren!

Aus kommunikationstheoretischer Sicht läßt sich das Fremde am besten mit dem Unvertrauten beschreiben. Die Unvertrautheit bezieht sich hier nicht nur auf etwas Äußeres. Sie betrifft vor allem die fremdkulturellen Handlungs- und Sprechhandlungsmuster. Mit dem letzteren meine ich Sprachroutinen, Gesprächsverhalten und die Fremdsprachenkompetenz selbst. Das Fremde, das Unvertraute, bezeichnet also ,jenes noch-nicht-Erfahrene, das unser Handlungspotential in Frage stellt" (vgl. Boesch, 1996:90). Das Fremde beinhaltet somit einen gewissen „Grad an Unsicherheit“ (ebd.). Unsere kommunikativen Orientierungsmöglichkeiten in der Fremde sind eingeschränkt, wenn wir mit der unbekannten Fremde zu tun haben. Fremd ist uns das unbekannte Fremde, wo eine erfolgreiche Kommunikation mit der Umwelt kaum möglich ist. Das würde allerdings als Antwort auf die Frage sehr trivial klingen, wenn man nicht eine sinnvolle Unterscheidung zwischen dem unbekannten und bekannten Fremden vornehmen würde. 
Wie verhält es sich nun zwischen dem bekannten und unbekannten Fremden und dem Eigenen?

\section{Das Fremde und das Eigene}

In unserem monokulturellen Alltag haben wir in der Regel selten Anlaß, uns mit fremden Kulturen auseinanderzusetzen. Es sei denn, wir haben einen intensiven Kontakt mit nicht angepaßten Ausländern. Unsere Wahrnehmung des Fremdkulturellen bleibt allerdings meist ethnozentrisch: d. h. wir befinden uns in Paradigmen unserer eigenen Welt und messen das Fremde sozusagen „mit der eigenen Latte“. Das Fremde kann uns interessant, eigenartig und sogar komisch vorkommen, aber auf alle Fälle bleibt es erklärungsbedürftig. Wir generalisieren gern das Fremde, füllen unsere Wissenslüicken über das Fremde mit Deutungen aus und greifen oft auf die Stereotypisierung zurïck, um unsere Orientierung nicht zu verlieren, und um vor allem unser eigenes Verhalten nicht ändern zu müssen. Und schließlich „gilt das Fremde als verstanden, wenn es in die vertrauten Kategorien übersetzt ist" (Duerr, 1978:152).

Aus der vertrauten Umgebung des Heimatlandes heraus wird das weite, nicht erlebte Fremde gern exotisiert, und meistens ein positives aber auch ein negatives Bild kreiert. (Diese Bilder kennen wir aus der romantischen Literatur, aus den Märchen und aus der Touristikbranche). Die Exotisierung und Sentimentalisierung des Fremden sind schlicht „idealisierender Projektionsrahmen von Hoffnungen und Visionen persönlichen Glïicks und der Selbstverwirklichung" (Wierlacher, 1993:76). Die idealisierte Fremde, die mit der fremdkulturellen Wirklichkeit und ihrem Alltag wenig zu tun hat, ist für das Leben und Arbeiten in einem fremden Land kaum hilfreich, wenn nicht sogar schädlich. Es gibt genügend Studien über Auslandseinsätze von Fach- und Führungskräften aus der Wirtschaft, in denen beschrieben wird, wie bei betroffenen Personen die idealistisch-positive Einstellung zum Fremden während des Auslandsaufenthalts ins Gegenteil umschlägt (s. z. B. Deller, 1996).

Der Kontakt mit der fremden Kultur in der Fremde ist wesentlich anstrengender als zu Hause. Es handelt sich hier nicht um touristische Reisen in ferne Länder. Uns geht es hier vor allem um einen längeren Arbeitsaufenthalt im Ausland, wo die Bemühung im Vordergrund steht, den Anschluß an die fremdkulturelle Gesellschaft zu finden, um dort den Arbeitsaufgaben erfolgreich nachgehen zu können. Zu der Auseinandersetzung mit dem Fremden kommt gezwungenermaßen die Auseinandersetzung mit dem Eigenen, was nicht unbedingt einfacher ist. Die bekannte Gegensätzlichkeit zwischen dem Fremden und dem Eigenen birgt viel Spannung in sich und kann zu jeder Zeit Grund für innere wie äußere Konflikte sein.

Das Eigene war schon immer zugänglich und durchschaubar, weil es an bekannte Regeln gebunden war. Es beinhaltete zwar auch „Zwänge, Pflichten und Einschränkungen der Freiheit" (vgl. Boesch, 1996:88). Aber man wußte, wie man zu handeln hatte und welche Verstöße wie bestraft werden. Nun beginnt man das Eigene mit einem fremden Blick zu betrachten, über das Selbstverständliche nachzudenken. Man muß für das Eigene geradestehen und dazu noch mit dem zurechtkommen, was einem in der fremden Kulturgemeinschaft als vermeintlich Eigenes zugeschrieben wird. Ich meine hier die Stereotypen und Vorurteile, mit denen man unbedingt in der Fremde konfrontiert wird und die man vorher oft gar nicht kannte. Und wenn es in der Fremde ganz schwierig wird, dann idealisieren wir auch das Eigene. Sobald man den Zustand erreicht hat, in dem man über das Eigene reflektieren kann, beginnt es, die Statik zu verlieren. Dann wehren wir uns emotional gegen das Fremde, oder einige Aspekte der eigenen Identität werden fraglich. Es wird etwas fremder, etwa zum fremden Eigenen. Dieses Befinden wurde von Veit (1987:43) mit einer schönen Metapher beschrieben, indem er vom „Schwebezustand“ in der Fremde spricht.

Nun haben wir nicht nur mit dem bekannten und unbekannten Fremden, sondern auch mit dem fremden - auf der ersten Stufe der Akkulturation in der Fremde womöglich auch mit einem unbekannten - Eigenen zu tun! Kann man das noch richtig auseinanderhalten? Gibt es überhaupt ein Gleichgewicht zwischen dem Fremden und dem Eigenen? Eindeutig positive Antworten auf die beiden Fragen wären sicherlich eher Idealisierungen. Viel wichtiger scheinen hier zunächst die Wege zu sein, auf denen wir uns das Fremde aneignen können.

\section{Der Weg der Aneignung des Fremden}

Zunächst muß hier hervorgehoben werden, daß der Kontakt mit dem Fremden immer ein Prozeß ist, und zwar ein innerer Entwicklungsprozeß. Der anfängliche Kontakt fuihrt immer zu einer stärkeren Abgrenzung. Als Beispiel brauchen wir nicht mal ferne Länder zu bemühen; ein kurzer Blick in die jüngste deutsche Vergangenheit erinnert uns an die Probleme der Verständigung zwischen West- und Ostdeutschen. Die anfängliche Fremdheit wurde in den Bezeichnungen „Wessi“ und "Ossi“ - mit allen dazugehörenden Konnotationen auch sprachlich festgehalten.

Eine Abgrenzung zwischen Kulturgemeinschaften, die unterschiedliche Sprachen sprechen, ist allerdings eindeutiger. Die Grenze kann man sogar „hören“. Die sprachlichen Grenzen sind im gewissen Sinne zugleich auch kulturelle Grenzen, auch wenn die Rede von intrakulturellen Subklassifizierungen (z. B. Soziolekte, Dialekte u. ä.) ist. Der Realitätssinn soll uns davor bewahren, die kulturellen sowie sprachlichen Grenzen zu nivellieren und die Kulturunterschiede bagatellisieren zu wollen, im Gegenteil, sie sollen erhellt, begriffen und geachtet werden. Die Kulturen müssen sich auch voneinander abgrenzen, um tradierbar zu bleiben (vgl. Assmann, 1990). Aus einer lerntheoretischen Perspektive können die Kulturen sinnvoll als „differierende Spielsysteme verstanden und verständlich gemacht werden“, meint Wierlacher (vgl. 1993:52). 
Es gibt mehrere Möglichkeiten, fremdkulturelles Wissen zu erwerben. Die eine ist der kognitive Weg zu diesem Wissen. Um das Fremde richtig zu verstehen, müssen wir uns mit ihm selbstverständlich zunächst beschäftigen. Durch die Erforschung des Fremden verwandelt es sich allmählich in das bekannte Fremde, mit dem wir dann angemessen umgehen können. Die kulturwissenschaftliche Xenologie versteht unter Aneignung des Fremden „immer auch einen Modus selbstkritischer Veränderung des Eigenen“ (Wierlacher, 1993:110). Der „selbstkritischen Veränderung " und somit Verfremdung des Eigenen sollte man nicht mit Vorbehalt begegnen. Sie ist eine der Voraussetzungen für interkulturelles Lernen. Sie ist auch der eigentliche „kulturelle Mehrwert“ (ebd. S. 103), der u. a. dank dem fremden Blick auf das Eigene entsteht. Der Fremdblick ist als Lernschritt produktiv, weil er das richtige Verstehen des Fremden erst ermöglicht.

Die Fremdheit des Eigenen ist u. E. eine Zwischenstufe in dem dynamischen Prozeß des interkulturellen Lernens. Das fremde Eigene wird allmählich wieder zum bekannten/vertrauten Eigenen, wenn man in der Fremde mit dem (nun bekannten) Fremden klarkommt. Weder soll das Eigene dem Fremden noch das Fremde dem Eigenen geopfert werden. Das Fremde und das Eigene soll in ihren Grenzen akzeptiert werden, denn jede kulturelle und kommunikative Assimilation hat ihre Grenzen. Nur durch eine gewisse Distanz zum Fremden läßt sich das Gleichgewicht bewahren. Allerdings, eine scharfe Grenze zwischen dem bekannten Fremden, das z. T. zum Eigenen geworden ist und dem „ursprünglich“ Eigenen, läßt sich kaum ziehen. Deshalb passiert es oft, daß bei Rückkehr in die Heimat nach einem längeren Aufenthalt im Ausland das Gleichgewicht für eine gewisse Zeit - bis man sich wieder integriert hat - gestört ist. (Zu Reintegrationsproblematik s. Winter, 1996).

Es wäre realitätsfremd zu behaupten, daß die interkulturelle Kommunikation reibungslos funktionieren würde, wenn man das Wissen über das Fremde allein auf kognitivem Wege erworben hat. Das affektive Lernen wird die Begegnung mit der Fremde immer begleiten. Hier stößt man allerdings eher an die Grenzen interkulturellen Verstehens, die in allen Fällen existieren. Eine qualifizierte Schulung kann jedoch den Betroffenen auf die Überraschungen im Ausland besser vorbereiten. Aber der affektive Lernweg allein kann sehr dornig werden.

Unbestritten ist es auch, daß die Kenntnis der Landessprache eine „Eintrittskarte“ in die neue Gesellschaft ist. Bereits beim Erlernen der Fremdsprache erfolgt eine enorme Umstellung des Denkens, denn mit der Fremdsprache wird auch ein Teil kulturellen Wissens erworben. Auch umgekehrt gilt, daß die Kenntnis fremder Kultur auf das Erlernen der entsprechenden Sprache eine positive Auswirkung hat.

\section{Wie fremd ist uns das Fremde?}

Nun zum Schluß noch eine weitere Antwort auf meine Frage. Ob und wie lange uns das Fremde fremd bleibt, bestimmen wir durch unsere eigene „Definition“ des Fremden. Die Antwort hängt in gewissem Sinne auch von uns selbst ab:
- von unserem Willen, das Fremde begreifen zu wollen,

- von unserer Fähigkeit, das Fremde verstehen zu können, und nicht zuletzt

- von unserer Neugier auf das Fremde, d. h. von unserem Wunsch, das Fremde überhaupt kennenzulernen.

Auch eine routinierte Art, mit bestimmten Fremden umzugehen, sollte nicht zum Verlust des Interesses am Fremden führen.

\section{Fußnoten}

1 Im Duden (Bd. 5, Das Fremdwörterbuch, 1994) wird die Xenologie immer noch als Okkultismus erklärt!

2 Diese Oppositionsbegriffe werden in den einschlägigen Publikationen gern als Untertitel genommen, vgl. z. B. Rothbucher, H.Wurst, F. (1989) und Schäffler, O. (1991).

\section{Literatur}

Assmann, Aleida/Assmann, Jan (1990): Kultur und Kulturkonflikt. Aspekte einer Theorie des unkommunikativen Handels. In: Assmann, J./Harth, D. (Hrsg.): Kultur und Kulturkonflikt, S. 11-48, Frankfurt a. M.

Boesch, Ernst E. (1996): Das Fremde und das Eigene. In: Thomas, Alexander (Hrsg.): Psychologie interkulturellen Handels, S. 87-105, Göttingen, Bern u. a.

Deller, Jürgen (1996): Interkulturelle Eignungsdiagnostik. In: Thomas, Alexander (Hrsg.): Psychologie interkulturellen Handels., S. 283-310, Göttingen, Bern u. a.

Duerr, Hans Peter (1978): Traumzeit. Über die Grenze zwischen Wildnis und Zivilisation. Frankfurt a. M.

Rothbucher, Heinz/Wurst, Franz (1989): Wir und das Fremde. Faszination und Bedrohung. Salzburg.

Schäffter, Ortfried (Hrsg.) (1991): Das Fremde. Erfahrungsmöglichkeiten zwischen Faszination und Bedrohung. Opladen.

Veit, Walter (1987): Der Schwebezustand. In: Grassi, E./ Schmale, H. (Hrsg.): Anspruch und Widerspruch. S. 4347. München.

Wierlacher, Alois (1993): Kulturwissenschaftliche Xenologie. Ausgangslage, Leitbegriffe und Problemfelder. In: Ders. (Hrsg.): Kulturthema Fremdheit. Leitbegriffe und Problemfelder kulturwissenschaftlicher Fremdheitsforschung, S. 19-112, München.

Winter, Gerhard (1996): Reintegrationsproblematik: Vom Heimkehren in die Fremde und vom Wiedererlernen des Vertrauten. In: Thomas, Alexander (Hrsg.): Psychologie interkulturellen Handels. S. 365-381, Göttingen, Bern u. a.

\section{Verfasserin}

\section{Dr. phil. Olga Rösch}

Technische Fachhochschule Wildau

Leiterin des Sprachenzentrums und des Instituts

für interkulturelle Kommunikation

Bahnhofstraße

15745 Wildau

Tel. +49 (0) 3375 -508367 und 508348 